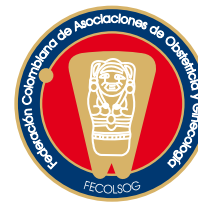

EDITORIAL

\title{
LA MORBILIDAD MATERNA EXTREMA: UNA OPORTUNIDAD PARA APRENDER DE EXPERIENCIAS EXITOSAS EN EL MANEJO DE LA COMPLICACIÓN OBSTÉTRICA
}

\section{Extreme maternal morbidity: an opportunity to learn from successful experiences in handling obstetric complications}

John Jairo Zuleta, M.D., MSc*

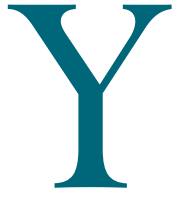
a es clásica la frase de Marta Gonzales de Paco, líder indígena aymará de El Alto, Bolivia: "Todas las mujeres que dan vida caminan por los andamios de la muerte".

Es labor de todo aquel que esté en contacto con una gestante, no solo del sector de la salud sino de todos los estamentos de la sociedad, poner todos los medios para disminuir al máximo los riesgos de este camino y son los obstetras los llamados a dictar las directrices para que esto se cumpla, mejorando la calidad del cuidado obstétrico.

La razón de mortalidad materna ha sido tradicionalmente el indicador empleado para evaluar la salud materna y la calidad de este cuidado, indicador que además refleja de manera directa las condiciones de desarrollo de los países y sus regiones. La integración del estudio de la morbilidad obstétrica al de la mortalidad incrementa la oportunidad de identificar los factores que contribuyen a ese continuum desde el embarazo sin complicaciones hasta la muerte y permite tomar correctivos de una manera más oportuna. En ese recorrido no solo pueden quedar familias sin la presencia de la madre, con todas las repercusiones sociales y económicas que esto implica, sino que

\footnotetext{
* Profesor Asociado. Departamento de Obstetricia y Ginecología. Universidad de Antioquia.

Correo electrónico: jjzuleta@une.net.co
}

también pueden sobrevivir un número aún mayor de mujeres con secuelas temporales o definitivas, con la consecuente pérdida económica y de calidad de vida para la sociedad y para la superviviente. La Organización Mundial de la Salud calcula que por cada muerte materna en Latinoamérica, 135 mujeres presentan enfermedades relacionadas con la gestación y aproximadamente 30 quedan con secuelas.

A pesar de las relativamente altas razones de mortalidad de nuestro país, el número absoluto de muertes por institución es bajo, por lo tanto se requiere un indicador de calidad de atención diferente a la mortalidad. La medición y evaluación de la morbilidad obstétrica extrema puede ser una medida más sensible en estos contextos y el punto de partida es la cuantificación de la magnitud del problema. El hecho de contar con esta medición permite conocer cuántas mujeres requieren cuidado obstétrico esencial e integral, las principales amenazas para la salud materna y los servicios de soporte que se requieren más frecuentemente. Igualmente, se obtiene la información necesaria para implementar, organizar y evaluar los programas que se requieren para la búsqueda de la maternidad segura. En los últimos años se ha dado un incremento en el interés de diferentes grupos en el país por trabajar estos temas, evidenciado tanto en los congresos de la especialidad como en las publi- 
caciones. El artículo central del actual número de la revista es un ejemplo de esta tendencia, práctica que se debe generalizar y profundizar para aportar a la salud de nuestras mujeres.

El artículo de los doctores Morales, Martínez y Cifuentes presenta la experiencia en un hospital universitario donde se destaca el efecto de la preeclampsia y la hemorragia obstétrica, como las complicaciones obstétricas extremas más frecuentes, lo cual concuerda con las principales causas de muerte materna en el país. Este tipo de análisis es el punto de partida para obtener una mayor comprensión del problema. Después de hacer una primera aproximación mediante la descripción de la situación, es decir, conocer quiénes y de qué se están complicando, tal como lo hacen los autores, el siguiente paso es identificar los factores determinantes que están influyendo para su presentación, pero ante todo, reconocer cuáles fueron las conductas o las condiciones que impidieron que estas madres murieran, con el fin de aplicar este conocimiento en la atención de futuras mujeres.

Los determinantes de la mortalidad y la morbilidad no son exclusivos de la atención dentro del sistema de salud ni del momento de la atención final de la complicación. Se encuentran problemas en la comunidad, en el sector de la salud y en otros sectores de la sociedad que influyen directamente en la complicación o de la muerte. En el estudio de la mortalidad se acude a los familiares y allegados para obtener información adicional de estos factores. La entrevista directa a las mujeres que padecieron la complicación ofrece ventajas: la información es oportuna, más confiable y adicionalmente el ambiente de la entrevista es más favorable, al no tener un desenlace fatal. Igualmente es más fácil obtener información adicional del personal médico, con una menor carga emocional porque se impidió una muerte. Por último, el aspecto más importante: se pueden detectar aspectos positivos que fueron los que evitaron el desenlace fatal y se cambia el enfoque del análisis de la muerte, generalmente asumido como punitivo, por otro más proactivo, con oportunidad de identificar y resaltar aspectos positivos a replicar. Cumplir esto trae a la realidad la propuesta de las doctoras Carini Ronsmans y Veronique Filippi ${ }^{1}$, dos pioneras en estos estudios: aprender de las supervivientes, que es la esencia del análisis de la morbilidad obstétrica extrema.

\section{REFERENCIAS}

1. Ronsmans C, Filippi V. Reviewing severe maternal morbidity: learning from survivors of life-threatening complications. En: World Health Organization. Beyond the numbers. Reviewing maternal deaths and complications to make pregnancy safer. Geneva; 2004. p. 103-23. 